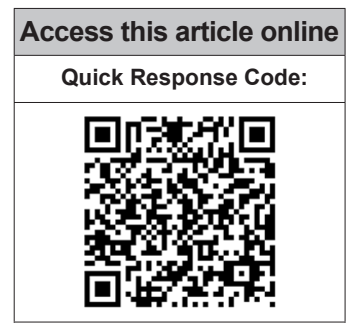

Website:

www.jponline.org

DOI:

10.4103/JLP.JLP_106_19
Department of Pathology, ICMR-National Institute for Research in

Environmental Health, Bhopal, Madhya Pradesh, ${ }^{1}$ Department of Pathology, PGIMER and Dr. RML Hospital, New Delhi, India

Address for correspondence: Dr. Arvind Ahuja, Department of Pathology, PGIMER and Dr RML Hospital, New Delhi - 110 001, India.

E-mail: drarvindahuja@ gmail.com

Submission: 20-06-2019 Accepted: 07-11-2019

\title{
Immunohistochemical expression of Ki-67, p53, and CD10 in phyllodes tumor and their correlation with its histological grade
}

\author{
Swasti Shubham, Arvind Ahuja ${ }^{1}$, Minakshi Bhardwaj ${ }^{1}$
}

\begin{abstract}
:
CONTEXT: Phyllodes tumors (PTs) are the fibroepithelial neoplasms of the breast. Histologically, PTs are divided into three subgroups according to their clinicopathological behavior: benign, borderline, and malignant. It is at times difficult to ascertain the grade of PT on morphological criteria alone, especially borderline PT may be at times difficult to distinguish from its benign or malignant counterparts.
\end{abstract}

AIMS: This study was undertaken to evaluate an immunohistochemical panel of Ki-67, p53, and CD10 in PT and to determine their expression in PT in correlation with its grade.

SETTINGS AND DESIGN: This was a retrospective study.

SUBJECTS AND METHODS: The study included six malignant, six borderline, and twelve benign PT. Expressions of Ki-67, p53, and CD10 were evaluated on all the 12 cases and compared in these three categories.

STATISTICAL ANALYSIS USED: Chi-square test was applied, and $P<0.05$ was taken as statistically significant.

RESULTS: Stromal expression of Ki-67 and p53 between the benign and borderline/malignant group showed a statistically significant difference. Neither CD10 expression nor epithelial expressions of Ki-67 and p53 were found significant. Periepithelial accentuation of Ki-67 and p53 immunostaining was noted in all positive cases.

CONCLUSIONS: Ki-67 labeling index and p53 immunostaining can be a useful adjunct to determine the grade in difficult cases. However, no single immunomarker can reliably distinguish between benign and borderline phyllodes in all cases.

Key words:

Benign, borderline, immunohistochemical, malignant, phyllodes, stromal cellularity

\section{Introduction}

Dhyllodes tumors (PTs) are the 1 fibroepithelial neoplasms of breast. PTs are relatively uncommon and comprise $<1 \%$ of all breast neoplasms. However, they are clinically important because of their unpredictable behavior, frequent recurrences following surgical resection and malignant transformation. ${ }^{[1,2]}$ According

This is an open access journal, and articles are distributed under the terms of the Creative Commons Attribution-NonCommercial-ShareAlike 4.0 License, which allows others to remix, tweak, and build upon the work non-commercially, as long as appropriate credit is given and the new creations are licensed under the identical terms.

For reprints contact: reprints@medknow.com to some authors, the biologic behavior of this tumor cannot be reliably predicted using only morphological features. ${ }^{[3,4]}$ Malignant progression has been observed even in initially benign appearing PT, which involves the stromal component of the tumor. This frequency of malignant progression has been reported to be $5 \%-30 \%$ of all PT by various studies. ${ }^{[5]}$

Histologically, PTs are divided into three subgroups: benign, borderline,

\footnotetext{
How to cite this article: Shubham S, Ahuja A, Bhardwaj M. Immunohistochemical expression of Ki-67, p53, and CD10 in phyllodes tumor and their correlation with its histological grade. J Lab Physicians 2019;11:330-4.
} 
and malignant. This classification reflects their clinic-pathological behavior and is based on the following histological criteria: (i) degree of stromal cellularity, (ii) stromal cytological atypia, (iii) mitotic activity, (iv) stromal overgrowth, and (v) the type of tumor margins (infiltrating/pushing). ${ }^{[6-8]}$ According to Tan et al., the relative incidence of benign, borderline, and malignant PT was $74.6 \%, 16.1 \%$, and $9.3 \%$, respectively, and the recurrence rate of PT was $12.8 \%$. They found that recurrence was related to the grade, atypia, cellularity, and permeative borders of the tumors. ${ }^{[9]}$ However, sometimes, it is challenging for the pathologist to ascertain the grade of PT on morphological criteria alone. Borderline PT may be difficult to distinguish from its benign or malignant counterparts. Due to the low incidence of this neoplasm, especially the borderline and malignant tumors, these features have not been adequately studied. In the past decade, few studies have highlighted the role of immunohistochemistry (IHC) panels to aid in the categorization of PTs. The aim of this study was to evaluate an IHC panel of Ki-67, p53, and CD10 expression in PT in correlation with its grade.

\section{Subjects and Methods}

The present study is a retrospective study which included six malignant and six borderline and twelve cases of benign PT diagnosed between January 2010 and December 2015. All tumor specimens were fixed in $10 \%$ buffered formalin and embedded in paraffin. Five $\mu \mathrm{m}$ thick sections placed on glass slides and stained with hematoxylin and eosin ( $\mathrm{H}$ and $\mathrm{E})$. All cases were reviewed and all the diagnoses were reconfirmed. Grading of cases as benign, borderline and malignant was done on the basis of WHO criteria. Stromal overgrowth has been defined as marked stromal proliferation so that the epithelial elements are absent in at least one low power field $(\times 40) \cdot{ }^{[1]}$ Secondary and/or additional features were noted. Table 1 shows the criteria used in the present study for grading the PTs.

After confirmation of diagnosis on $\mathrm{H}$ and E-stained sections, $5 \mu \mathrm{m}$ was placed on polylysine-coated slides. IHC staining of Ki-67 (DAKO), p53 (DAKO), and CD10 (DAKO) was performed. Ki-67 and p53 were evaluated in both epithelial and stromal component. CD10 was evaluated in the stromal component. For Ki-67, percentage positive nuclei were counted in 5 high power fields (hot-spots). Ten percent or higher nuclear staining was considered significant. Nuclear positivity for p53 was graded as low, intermediate, or high intensity as compared to control. A positive result was defined as unequivocal staining of $10 \%$ or more cells. Membranous and cytoplasmic positivity for CD10 was also graded as low, intermediate, or high intensity as compared to control. Intermediate- to high-intensity staining of $>20 \%$ of stromal cells was considered positive. The results were tabulated, and significance was calculated $(P<0.05)$ using the Chi-square test. Lymph nodes were used as positive controls for Ki-67 and CD10. Known positive cases of urothelial carcinomas were used as positive control for p53. Myoepithelial cells of ducts also acted as internal positive control for CD10.

\section{Results}

\section{Demographics}

Age of the patients ranged from 25 to 72 years. The mean age for patients with benign, borderline, and malignant PT was 36.0 years, 38.5 years, and 48 years, respectively. The mean diameter of the tumor was $7 \mathrm{~cm}$ for benign, $12.5 \mathrm{~cm}$ for borderline, and $17.5 \mathrm{~cm}$ for the malignant group.

\section{Histomorphology}

Microscopically, all six malignant PT showed high stromal cellularity, marked cellular pleomorphism, >10 mitoses/10 high power field, infiltrative margin, and stromal overgrowth. Two of these cases were composed predominantly of stromal component with only occasional duct representing the epithelial component. Heterologous sarcomatous element was identified in one case which showed an area of chondroblastic osteosarcoma. Other epithelial changes noted were epithelial hyperplasia and cystic change in one case each.

\section{Immunohistochemistry}

IHC panel applied on benign, borderline, and malignant PT was compared [Tables 2 and 3].

\section{Ki-67}

All malignant PT showed Ki-67 nuclear positivity in $15 \%-40 \%$ stromal cells while all benign showed only $2 \%-6 \%(<10 \%)$ nuclear positivity in stromal cells. Among borderline PT, four out of six cases showed Ki-67 staining in $10 \%-20 \%$ of stromal cell nuclei [Figure 1] while the two cases showed $<10 \%$ positive nuclei. Overall, the percentage of positive nuclei in borderline PT was intermediate between the malignant and benign cases. In contrast to stromal cells, the epithelial cells did not show any significant difference in Ki-67 expression. The $\mathrm{Ki}-67$ staining was found to be statistically significant in differentiating benign PT from borderline/malignant PT with a $P=0.0002$. On comparison of borderline and benign PT cases, Ki-67 showed a statistically significant difference with a $P=0.0091$.

p53

Stroma of 4 out of 6 malignant PT, three out of six borderline PT and only one out of twelve benign PT were positive for p53 [Figure 2]. Three positive malignant PT showed strong staining intensity while the only positive benign PT showed moderate staining intensity. Rest of 
Table 1: WHO grading criteria for phyllodes tumors

\begin{tabular}{llll}
\hline Criteria & Benign & Borderline & Malignant \\
\hline $\begin{array}{l}\text { Stromal cellularity } \\
\text { and atypia }\end{array}$ & Minimal & Moderate & Marked \\
$\begin{array}{l}\text { Stromal overgrowth } \\
\text { Mitoses/10 high }\end{array}$ & Minimal & Moderate & Marked \\
$\begin{array}{l}\text { power fields } \\
\text { Tumor margins }\end{array}$ & $\begin{array}{l}\text { Well } \\
\text { circumscribed } \\
\text { with pushing } \\
\text { tumor margins }\end{array}$ & $\begin{array}{l}\text { Zone of } \\
\text { microscopic } \\
\text { invasion around } \\
\text { tumor margins }\end{array}$ & $\begin{array}{l}\text { Infiltrative } \\
\text { tumor }\end{array}$ \\
\hline
\end{tabular}

Table 2: Comparison of immunohistochemistry markers expression in borderline and malignant Phyllodes tumors with benign Phyllodes tumors

\begin{tabular}{lccccccc}
\hline & \multicolumn{3}{c}{ Malignant/borderline } & \multicolumn{3}{c}{ Benign } & $P$ \\
\cline { 2 - 6 } & Total & Positive & Negative & Total & Positive & Negative & \\
\hline Ki-67 & 12 & 10 & 2 & 12 & 0 & 12 & 0.0002 \\
p53 & 12 & 7 & 5 & 12 & 1 & 11 & 0.0093 \\
CD10 & 12 & 8 & 4 & 12 & 7 & 5 & 0.6732 \\
\hline
\end{tabular}

Table 3: Comparison of immunohistochemistry markers expression in borderline Phyllodes tumors with benign Phyllodes tumors

\begin{tabular}{lccccccc}
\hline & \multicolumn{3}{c}{ Borderline } & \multicolumn{3}{c}{ Benign } & $P$ \\
\cline { 2 - 7 } & Total & Positive & Negative & Total & Positive & Negative & \\
\hline Ki-67 & 6 & 4 & 2 & 12 & 0 & 12 & 0.0091 \\
p53 & 6 & 3 & 3 & 12 & 1 & 11 & 0.0450 \\
CD10 & 6 & 4 & 2 & 12 & 7 & 5 & 0.7324 \\
\hline
\end{tabular}

the positive PTs showed an intermediate intensity of p53 expression. The percentage of positive nuclei ranged from $10 \%$ to $25 \%$ but did not show any correlation with grade. Staining for p53 was statistically significant in differentiating between benign and borderline/ malignant $\mathrm{PT}$ as well as between benign and borderline PT with a $P=0.0093$ and 0.0450 respectively. As seen in the case of Ki-67, the significant difference in staining was observed in the nuclei of stromal cells but not in the epithelial cells.

\section{CD10}

Four of six malignant and as well as borderline PT and seven of twelve benign PT were positive for CD10. The staining intensity was moderate to strong in all positive cases. However, unlike Ki-67 and p53, the difference between the malignant/borderline cases and benign cases was not statistically significant [Figure 3].

\section{Discussion}

PT is characterized by uncertain biological behavior with potential for recurrences as well as malignant progression. These tumors show a wide range of clinicopathological features ranging from benign lesions indistinguishable from fibroadenoma to malignant forms overlapping with fibrosarcoma. In malignant PT,

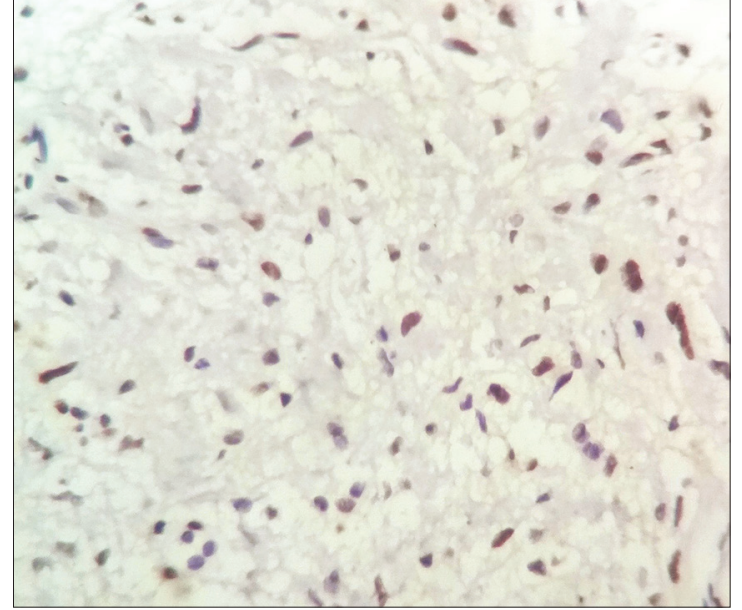

Figure 1: Nuclear expression of Ki-67 immunostain in a case of borderline phyllodes tumors $(\times 400)$

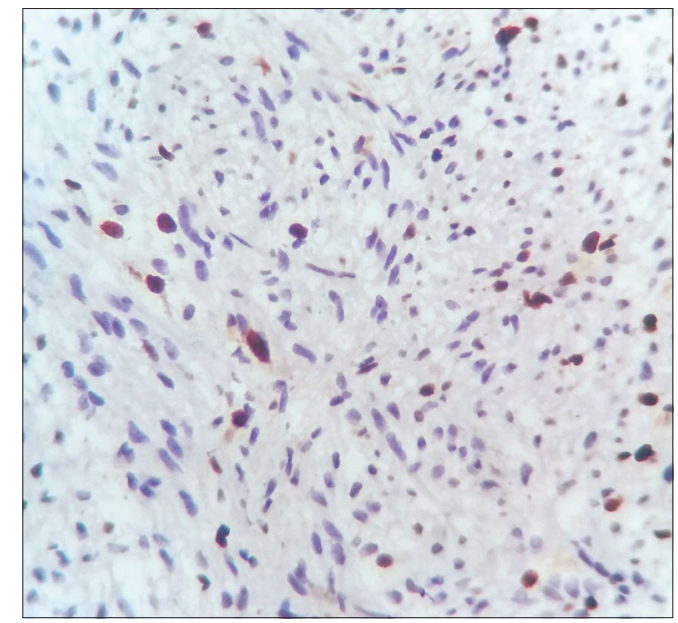

Figure 2: Nuclear expression of p53 immunostain in a case of borderline phyllodes tumors $(\times 400)$

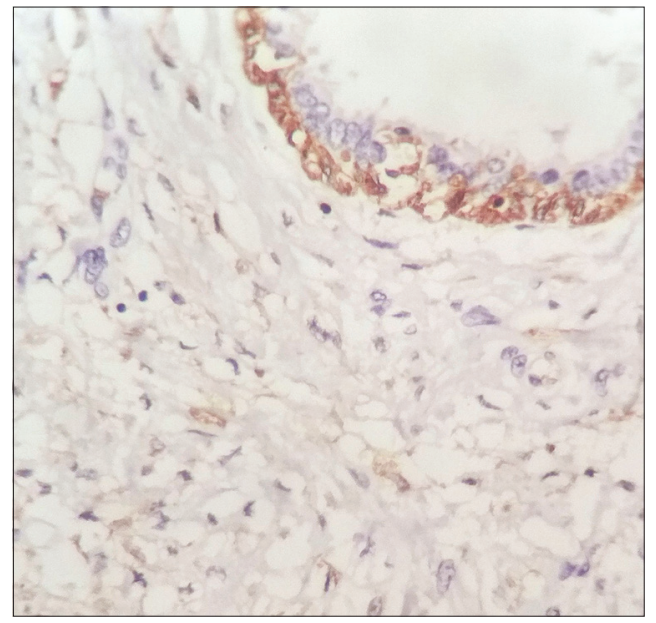

Figure 3: CD10 negative borderline phyllodes tumors. The myoepithelial layer of mammary duct shows cytoplasmic positivity and acts as internal control $(\times 400)$

stroma undergoes transformation to sarcoma with an increase in mitotic and stromal overgrowth. Stromal- 
epithelial interaction is thought to be important in the development and malignant transformation of PT. ${ }^{[10]}$ The criteria used to determine malignancy in PT, therefore reflects its pathogenesis. Several clinical factors such as older age and larger lesions have been found to have a greater association with borderline and malignant PT. However, the interpretation of histological parameters used to grade PT is still somewhat subjective and does not strictly correlate with biological behavior. ${ }^{[1]}$

A number of IHC markers have been studied in PT for diagnosis and subtyping. The neoplastic component of PT is the stroma which is thought to arise from periductal stromal cells. This is supported by the findings that stroma of PT shows positive reaction for vimentin, a variable reaction for actin, CD34 and desmin, and a negative reaction for S-100 protein. Ultrastructurally, the stromal cells of PT are composed of cells with features of both fibroblasts and myofibroblasts. These stromal cells resemble the normal mammary stromal cells. ${ }^{[11]}$ In the study by Kaya et al., proliferating cell nuclear antigen and Ki-67 immunostaining did not reveal significant differences between PT and fibroadenoma. ${ }^{[5]} \mathrm{Ki}-67$, p53 and c-kit have been reported to be useful by some authors in distinguishing benign from malignant PT in diagnostically difficult cases. However, only c-kit was found useful in predicting recurrences in PT. ${ }^{[2,9]}$ Tse et al. found CD10 to be consistently positive in normal breast myoepithelial cells and CD10 may be a useful adjuvant in assessing the fibroepithelial neoplasms of the breast. ${ }^{[12]}$ No clinical or histological factor, either singly or in combination has been found to be useful in borderline PT or to reliably predict the outcome in every patient. Hence, there is an ongoing search for IHC and molecular markers which may prove to be useful to pathologists and clinicians for diagnosis and treatment. In the various studies discussed above, Ki-67, p53, and CD10 were found to correlate with the grade of PT most closely, even though the actual results of these studies varied widely. $[2,9,12-16]$

In the present study, the cellular proliferation marker Ki-67 was found to correlate with the grade of PT. 100\% of malignant and $66.66 \%$ of borderline PT was positive for Ki- 67 while $100 \%$ of benign PT was negative for the same. This difference of expression between malignant/ borderline and benign groups was statistically significant. Similar results were published by other researchers. $[2,13,14,16]$ In the literature, the $\mathrm{Ki}-67$ labeling index is $5 \%-25 \%$ in benign and $15 \%-100 \%$ in malignant PTs. ${ }^{[17]}$ In our series, this index was $1 \%-8 \%$ in benign PT, $6 \%-20 \%$ in borderline PT, and $15 \%-40 \%$ in malignant PT, showing an overlap between malignant and borderline cases. Similar results were reported by Kleer et al., who found slight overlap at high and low Ki-67 labeling indexes. ${ }^{[2]}$ According to Esposito et al. as well, mean Ki-67 labeling indexes for benign, borderline, and malignant PTs were 3.6\%, 26\%, and $32 \%$, respectively. ${ }^{[16]}$ Both these groups also found that clinical behavior and recurrences could not be predicted by Ki-67 labeling index.

p53 expression was noted in $66.66 \%$ of malignant, $50 \%$ of borderline and only $8.33 \%$ of benign PT which is in accordance to some previous studies that revealed high p53 expression rates associated with increasing tumor grade. ${ }^{[13,16]}$ As seen in the case of $\mathrm{Ki}-67$, the difference of expression between malignant/borderline and benign groups was statistically significant. Tan et al. also noted p53 immunohistochemical positivity in a proportion of luminal epithelial and myoepithelial cells in their series of PTs. ${ }^{[9]}$ Other reports have described p53 reactivity in the breast epithelium in conjunction with stromal staining. The finding of dual expression of p53 protein in the stroma as well as the epithelium of breast highlights the likelihood of an interaction between these two components. ${ }^{[3,18]}$ In the present study, though p53 staining was noted in the stroma and focally in ductal epithelial cells, the myoepithelial cells remained consistently negative.

CD10 is a myoepithelial marker in breast tissue. In normal breast, the stromal cells were found to be negative for CD10, but it was claimed to be positive in the stroma of breast malignancy and fibroepithelial lesions. ${ }^{[12,15]}$ Unlike Ki-67 and p53, we did not find any correlation between tumor grades and CD10 staining. It was positive in $33.33 \%$ of malignant PT, $100 \%$ of borderline PT and $58.33 \%$ of benign PT. No statistically significant difference was found between these groups, even though stromal cells in PT took up CD10 immunostaining to a variable degree.

In agreement with some previous studies, we found periepithelial accentuation of $\mathrm{Ki}-67$ and p53 immunostaining in all positive cases. ${ }^{[2,9]}$ This finding seems to corroborate the hypothesis that the "pool of proliferating cells" reside in the periepithelial or periductal region and that PT arises from periductal rather than intralobular stromal cells. ${ }^{[2]}$ In addition, both these markers showed a higher expression in the stromal component of malignant and borderline PT emphasizing that it is the stroma that undergoes malignant change. In our experience, malignant PT are often sarcoma-like in appearance and do not present difficulty in diagnosis on histological examination. The difficulty lies in the diagnosis of borderline PT and its distinction from benign cases. The interobserver variability in the diagnosis of borderline PT is high and affects further management. In such a scenario, Ki-67 and p53 immunostaining can serve as a useful adjunct.

Since this was a retrospective study, we could not correlate our results with clinical outcome and follow-up 
of the patient, which is a limitation of our study. In addition, the sample size is limited due to the rarity of malignant PT.

\section{Conclusions}

Ki-67 labeling index and p53 immunostaining can be useful adjuncts to determine the grade in difficult cases. However, no single immunomarker can reliably distinguish between benign and borderline phyllodes in all cases. Further studies are required to establish the correct combination of immunomarkers to distinguish between these two groups and also to accurately predict clinical behavior.

\section{Financial support and sponsorship Nil.}

\section{Conflicts of interest}

There are no conflicts of interest.

\section{References}

1. Tan PH, Jayabaskar T, Chuah KL, Lee HY, Tan Y, Hilmy M, et al. Phyllodes tumors of the breast: The role of pathologic parameters. Am J Clin Pathol 2005;123:529-40.

2. Kleer CG, Giordano TJ, Braun T, Oberman HA. Pathologic, immunohistochemical, and molecular features of benign and malignant phyllodes tumors of the breast. Mod Pathol 2001;14:185-90.

3. Dacic S, Kounelis S, Kouri E, Jones MW. Immunohistochemical profile of cystosarcoma phyllodes of the breast: A study of 23 cases. Breast J 2002;8:376-81.

4. Niezabitowski A, Lackowska B, Rys J, Kruczak A, Kowalska T, Mitus J, et al. Prognostic evaluation of proliferative activity and DNA content in the phyllodes tumor of the breast: Immunohistochemical and flow cytometric study of 118 cases. Breast Cancer Res Treat 2001;65:77-85.

5. Kaya R, Pestereli HE, Erdogan G, Gülkesen KH, Karaveli S.
Proliferating activity in differential diagnosis of benign phyllodes tumor and cellular fibroadenomas: Is it helpful? Pathol Oncol Res 2001;7:213-6.

6. Moffat CJ, Pinder SE, Dixon AR, Elston CW, Blamey RW, Ellis IO. Phyllodes tumours of the breast: A clinicopathological review of thirty-two cases. Histopathology 1995;27:205-18.

7. Ang MK, Ooi AS, Thike AA, Tan P, Zhang Z, Dykema K, et al. Molecular classification of breast phyllodes tumors: Validation of the histologic grading scheme and insights into malignant progression. Breast Cancer Res Treat 2011;129:319-29.

8. Tan PH, Thike AA, Tan WJ, Thu MM, Busmanis I, Li H, et al. Predicting clinical behaviour of breast phyllodes tumours: A nomogram based on histological criteria and surgical margins. J Clin Pathol 2012;65:69-76.

9. Tan PH, Jayabaskar T, Yip G, Tan Y, Hilmy M, Selvarajan S, et al. $\mathrm{P} 53$ and c-kit (CD117) protein expression as prognostic indicators in breast phyllodes tumors: A tissue microarray study. Mod Pathol 2005;18:1527-34.

10. Karim RZ, Scolyer RA, Tse GM, Tan PH, Putti TC, Lee CS. Pathogenic mechanisms in the initiation and progression of mammary phyllodes tumours. Pathology 2009;41:105-17.

11. Rosen PP. Rosen's breast pathology. $2^{\text {nd }}$ ed. Philadelphia: Lippincott Williams and Wilkins; 2001. p. 176-94.

12. Tse GM, Tsang AK, Putti TC, Scolyer RA, Lui PC, Law BK, et al. Stromal CD10 expression in mammary fibroadenomas and phyllodes tumours. J Clin Pathol 2005;58:185-9.

13. Kucuk U, Bayol U, Pala EE, Cumurcu S. Importance of P53, ki-67 expression in the differential diagnosis of benign/ malignant phyllodes tumors of the breast. Indian J Pathol Microbiol 2013;56:129-34.

14. Song JY, Yoon HK. Immunohistochemical phenotypes of phyllodes tumor of the breast. Korean J Pathol 2008 1;42:151-6.

15. Hussin H, Pailoor J, Cheng PS. The Role of CD10 Immunohistochemistry in the Grading Of Phyllodes Tumor of the Breast. J Interdiscipl Histopathol 2013;1:195-203

16. Esposito NN, Mohan D, Brufsky A, Lin Y, Kapali M, Dabbs DJ. Phyllodes tumor: A clinicopathologic and immunohistochemical study of 30 cases. Arch Pathol Lab Med 2006;130:1516-21.

17. Tse GM, Niu Y, Shi HJ. Phyllodes tumor of the breast: An update. Breast Cancer 2010;17:29-34.

18. Witte F, Hönig A, Mirecka J, Schauer A. Cystosarcoma phyllodes of the breast: Prognostic significance of proliferation and apoptosis associated genes. Anticancer Res 1999;19:3355-9. 